\title{
Favored location of a stabilizing screw for syndesmotic diastasis: Recommendation based on clinical and theoretical considerations
}

Chi-Chuan Wu ( $\nabla$ ccwu@mail.cgu.edu.tw)

Chang Gung Memorial Hospital, Chang Gung University https://orcid.org/0000-0003-3609-7986

\section{Wen-Ling Yeh}

Chang Gung Memorial Hospital

Po-Cheng Lee

Chang Gung Memorial Hospital

\section{Ying-Chao Chou}

Chang Gung Memorial Hospital

\section{Yung-Heng Hsu}

Chang Gung Memorial Hospital

Yi-Hsun Yu

Chang Gung Memorial Hospital

Research article

Keywords: Location, Outcomes, Screw, Syndesmotic diastasis

Posted Date: August 3rd, 2020

DOl: https://doi.org/10.21203/rs.3.rs-42576/v1

License: (9) (i) This work is licensed under a Creative Commons Attribution 4.0 International License.

Read Full License 


\section{Abstract}

Background Although ankle injuries are common, combined malleolar fractures and syndesmotic diastasis (SD) are relatively fewer. The optimal technique for syndesmotic stabilization has yet achieved consensus. The favored location of a stabilizing screw for SD was investigated clinically and theoretically.

Methods For the 10-year period, 63 consecutive adult patients with combined injuries were retrospectively studied. After malleolar fractures were internally stabilized with screws and plates, stress tests were performed to re-confirm syndesmotic instability. The diastatic syndesmosis was stabilized with cortical screws under image intensifier guidance. In the present study, only patients with one syndesmotic screw (55 patients) were enrolled and divided into the trans-syndesmotic (TR) or the supra-syndesmotic (SU) group. Clinical and functional outcomes between the two groups were compared. The favored location was speculated consequently.

Results Forty-eight patients were followed for at least one year (average, 1.8 years; range, 1.0-7.0 years). The TR group included 31 patients and the SU group, 17 patients. All clinical and functional comparisons were not statistically significant $(p>0.05)$.

Conclusions Although statistical comparison is insignificant, TR screw insertion possesses biomechanical and biological merits. Unable to reveal advantages of TR screw insertion in clinical and functional comparisons may be imputed to insufficient sample sizes and early screw removal. Practically, TR insertion of a screw with late removal (> 3 months) may be a better choice.

\section{Background}

Ankle injuries are common in the daily activity. Clinically, the majority of ankle injuries are not severe and non-surgical treatment techniques can usually achieve a success [1,2]. However, in some ankle injuries which normally comprise malleolar fractures and syndesmotic diastasis (SD) often require surgical treatment. Delayed or neglected treatment of these injuries may cause lifelong disabilities and patients cannot restore normal activity forever $[3,4]$.

Because the unfavorable anatomic structures of the ankle, huge tibia and small fibula constituting the syndesmosis, local stress transfer is very unsymmetrical $[5,6]$. The four stabilizing ligaments are often injured and even completely disrupted. Consequently, the stability of syndesmosis is lost and the talus dislocates immediately [5-8]. Once SD is associated with malleolar fractures, treatment becomes even more complicated. Until now, a globally satisfying classification for malleolar fractures with or without SD has yet been developed. In the literature, combined Weber and Lauge-Hansen classifications are often applied by many orthopedic surgeons [9-11]. They believe that the combined classifications may comprise the majority of ankle injuries requiring to pay attention. 
In the literature, SD may be treated with stabilizing cortical screws or the suture-button technique and each has unique advantages and disadvantages $[4,12]$. Despite that the latter has gradually achieved increasing supports recently, the former is still widely utilized because of its technical simplicity and high success rate. Even so, the optimal technique for screw insertion has yet achieved consensus: diameter, numbers, location, and purchased cortices [3,4,13]. Biomechanically, a stabilizing screw will sustain huge bending and rotational stresses in gait. The far is a screw from the point of load application (i.e. anteromedial aspect of the lateral malleolus), the bigger is its loading stresses [14]. In other words, a more proximally placed screw will sustain more bending and rotational stresses. The screw consequently breaks more possibly. Despite that the screw location is reported without affecting treatment outcomes in the literature, the mechanism is yet clarified in detail $[3,4,13]$. We hypothesized that the screw location should definitely affect treatment outcomes if biomechanical analysis is considered. The purpose of this retrospective study intended to verify whether screw location can indeed affect treatment outcomes and how it works based on clinical and theoretical considerations.

\section{Methods}

This study had been approved by Institutional Review Board of the authors' institution (IRB: 201900950B0).

From January 1, 2008 to December 31, 2017, 822 consecutive adult patients (> 18 years) who sustained malleolar fractures with or without SD and had been surgically treated at our institution were pursued for this study. The inclusion criteria for study were malleolar fractures combined with SD, acute injuries without prior surgical treatment, and complete medical records. The exclusion criteria for study were association with ipsilateral tibial fractures, old local deformities, and congenital or developmental anomalies. At last, 63 patients (44 men and 19 women; average of 33 years, range of 18-76 years) were matched for the study criteria. The causes of ankle injuries included motorcycle accident (27 cases), various sports injuries (11 cases), slide (8 cases), fall from stairs ( 6 cases), and various reasons (11 cases). Fifty-eight injuries were caused by loads from external rotation: 33 supination-external rotation and 25 pronation-external rotation (by Lauge-Hansen classification) [9-11]. Four cases were associated with type 2 or IIIA open fractures [15].

At the emergency service or outpatients' department (OPD), the patient's general condition was stabilized first. The surgeries were performed as early as possible (average, 5.2 days; rage, $0.5-14$ days).

\section{Surgical procedures:}

Under general anesthesia with endotracheal intubation, all patients were placed on the operating table in the supine position. A pneumatic tourniquet was routinely used and an image intensifier was prepared for all surgeries.

The contralateral uninjured ankle was used for control and the relative position of the lateral malleolus to the distal tibia was demonstrated on the fluoroscope [16]. The images were then transferred to the Picture 
Achieving and Communication System software (PACS; GE healthcare, Waukesha, WI, USA) immediately [17]. All malleolar fractures were explored and internal fixation with screws and plates (Synthes, Bettlach, Switzerland) was applied as indicated. The instability of syndesmosis was re-confirmed by external rotation and hook tests [7]. Consequently, closed reduction with pointed clamp holding on the distal fibula and distal tibia was performed [18]. The position of lateral malleolus related to the distal tibia was adjusted following the contralateral ankle images on the PACS. One or two cortical screws were inserted from the distal fibula to the distal tibia under the image intensifier guidance. The modes of screw insertion (numbers, diameter, location, and purchased cortices) completely depended upon the individual surgeon. After the wound was closed, a short leg splint was applied.

Postoperatively, all patients were encouraged to ambulate with protected weight bearing as early as possible. They were followed at the OPD at 4-6 weeks interval. The short leg splint was discontinued to use after 6 weeks. The decision of removal of syndesmotic screws was arranged by the individual surgeon. Removal of implants for malleolar fractures was advised after one year.

Clinical and radiographic healing processes were evaluated at the OPD. Fracture union was defined as clinically no pain and tenderness, and radiographically fracture gaps being vanished or the callus having bridged the gap [19].

The ankle function was evaluated based on American Orthopedic Foot and Ankle Society (AOFAS) scores [20]. Total scores were 100 points and included three items (pain, function, and alignment). An excellent grade was no fewer than 90 points, a good grade was no fewer than 80 points. A satisfactory grade included an excellent or good grade.

\section{Statistical analysis:}

A SPSS version 20 (SPSS Inc., Chicago, IL, USA) software was used for statistical comparison. $\mathrm{P}<0.05$ was considered statistically significant. The chi-square test was used for categorical data and the MannWhitney U test was used for numerical data.

\section{Results}

The syndesmotic screws in 56 patients were removed at an average of 2.2 months (range, 1.5-5.0 months) and seven patients did not.

Fifty-four patients were followed for at least one-year (85.7\%; average, 1.8 years; range, 1.0-7.0 years) and all malleolar fractures healed. Nine patients could not be contacted despite all possible efforts.

In the present study, location of the syndesmotic screw was planned for comparison. Consequently, only patients with one screw insertion were included. It included 31 trans-syndesmotic (TR) and 17 suprasyndesmotic (SU) cases. The follow-up rates were 86.1\% (31 / 36) and 89.5\% (17 / 19), respectively (Table 1). 


\section{Table 1}

Comparison of trans-syndesmotic and supra-syndesmotic insertions of a stabilizing screw for syndesmotic diastasis $(n=48)$

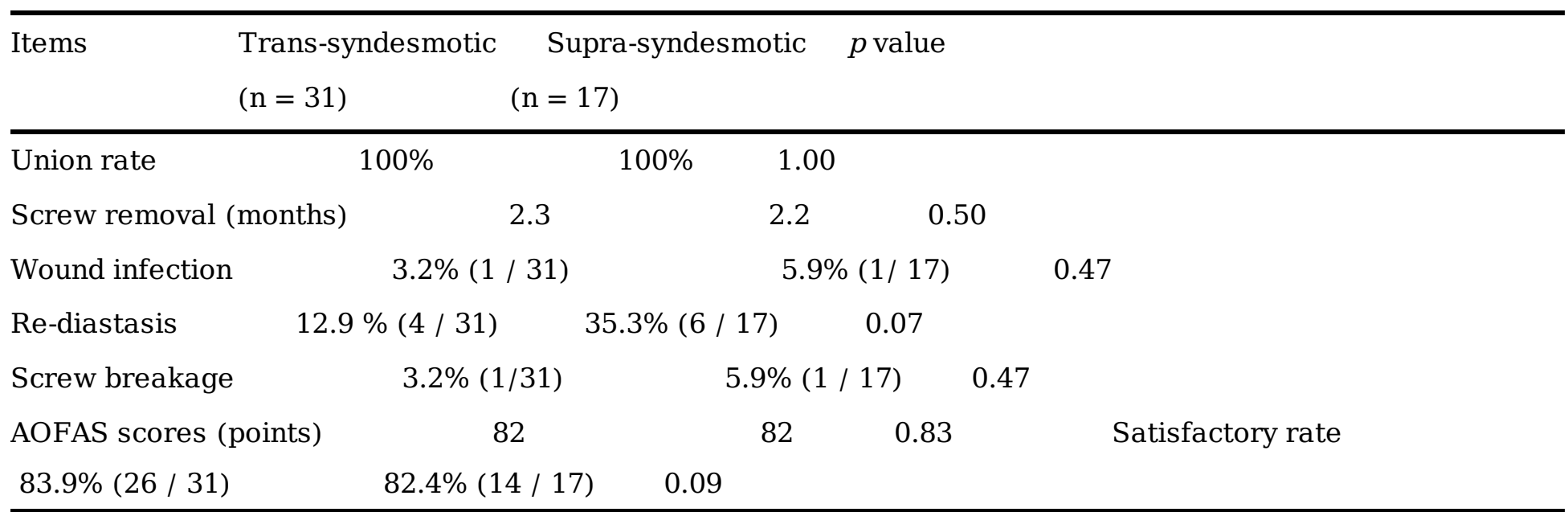

AOFAS, American Orthopedic Foot and Ankle Society

All malleolar fractures healed with a union rate of $100 \%$ (48/48) and the union time was fewer than 2 months (Figs. 1,2).

Complications included deep wound infection in two patients. Both wounds healed after once debridement and local care. Recurrent diastasis occurred in 4 (4 / 31, 12.9\%) TR and 6 (6 / 17, 35.3\%) SU cases ( $p=0.068$; post-hoc power $=0.45$; Figs. 3,4 ). Recurrent diastasis was defined as the tibiofibular clear space further separated beyond $2 \mathrm{~mm}$ by the comparison of post-operative and the latest anteroposterior radiographs [21]. The syndesmotic screw was removed at an average of 2.3 and 2.2 months, respectively. Screw breakage occurred in two patients and all were not removed early (2 / 5, 40\%; Figs. 5,6). Ankle osteoarthritis occurred in two patient and both were associated with an open fracture.

Ankle function was compared. The AOFAS score in TR group was an average of 82 points (range, $68-88$ points) and in SU group, an average of 82 points (range, 68-88, $p=0.83$ ).

A satisfactory ankle function was noted in 83.9\% (26 / 31) of TR group and $82.4 \%$ (14 / 17) of SU group $(p=0.89)$.

\section{Discussion}

Humans are bipedal animals and usually use one lower limb alternately in walking. During a gait, the body's center of gravity will move to the contralateral side and introduces huge bending and rotational moments [22]. An ideal implant used for stabilizing the fractured or dislocated lower limb must tolerate huge loads stereoscopically. 
Anatomically, the structures of the ankle are unfavorable for multi-directional movements. A large disparity of sizes between the distal tibia and the distal fibula introduces load transfer markedly unsymmetrically [5,6]. Additionally, the talus is simply allowed for plantar-dorsiflexion action [23]. As long as various directional loads are applied on the ankle, various complex ankle injuries will occur. Therefore, until now a simple, complete, and convincing classification for ankle injuries has yet been established. In the literature, combined Weber and Lauge-Hansen classifications are supported by many orthopedic surgeons [9-11]. They believe that the combined classifications can comprise the majority of ankle injuries related to the malleoli and syndesmosis.

Because reported articles with large sample sizes in treating the combined injuries are few, the optimal screw use in stabilizing the diastatic syndesmosis has yet achieved consensus [24,25]. In the present study, the favored screw location is enthusiastically pursued from clinical and theoretical considerations. Although the clinical results cannot support either superiority, TR screw insertion may be better based on theoretical comparison.

Biomechanically, the shorter is the lever arm, the less is the load moment $[14,26]$. When a cortical screw is inserted to maintain the syndesmotic stability, the screw becomes the fulcrum. The nearer is a cortical screw placed to the lateral malleolus (i.e. the point of force application), the less loaded is the screw. Therefore, TR insertion of a syndesmotic screw should be safer than SU insertion. Because the majority of stabilizing screws have been removed at an average of 2.2 months in the present study, screw breakage is few $(4.2 \%, 2$ / 48). Statistical comparison cannot therefore make a definite conclusion. If the syndesmotic screw is removed late or let it alone without removal, the majority of screws will fail. In 2005 , Kukreti et al. reported treatment of 36 patients with SD [27]. Nineteen cases were treated with SU screws and 17, TR screws. Syndesmotic screws were removed at 2 months and they found no difference in terms of clinical and radiological outcomes. The present study also achieved a comparable result.

Biologically, the four surrounding ligaments of the syndesmosis mainly gain the vascularity from the perforating branches of peroneal artery in the posterior compartment [28,29]. This site is slightly proximal to the upper border of the syndesmosis. SU insertion of a cortical screw will very possibly injure the perforating branches and hinder the ligament healing. Once the stabilizing screw is removed at 2.2 months for prevention of screw breakage, SD may recur. In the present study, SU insertion is found to increase the possibility of re-diastasis of the syndesmosis despite statistical insignificance $(35.3 \%$ versus $12.9 \% ; p=0.068$ ). The delayed healing of the four surrounding ligaments may be contributing factor.

In the literature, an unstable ankle caused by a diastatic syndesmosis will introduce a poor functional outcome [30,31]. Although SU insertion may have a higher incidence of syndesmotic re-diastasis $(35.3 \%$ versus $12.9 \%, p=0.068$ ) in the present study, the functional outcome of the ankle is still similar. The causes may be imputed to insufficient sample sizes (post-hoc power $=0.45$ ) and a short period of followup (an average of 1.8 years). 
Theoretically, poor ankle function after treatment of the combined injuries should be closely related to residual instability of the ankle $[31,32]$. In normal gait, the talus will be pushed by $2^{0}-6^{0}$ external rotation and $1.5 \mathrm{~mm}$ later shift at the stance phase [33]. If SD recurs, wear of articular cartilage will be speeded and osteoarthritis will be progressive. Clinically, this complication is critical but it is normally preventable as long as the joint stability is restored well [34]. The present study may therefore provide an important knack.

Situations of a syndesmotic screw affect the treatment outcomes are multiple. Screw diameter, location, numbers, and purchased cortices are considered without affecting the treatment outcomes $[3,4,13]$. However, the sample sizes reported in the literature are generally insufficient. All results are often contradicted without consensus. Purely making a conclusion from clinical studies seems to be less possible. The present study integrating clinical and basic concepts may speculate a relatively reasonable technique to improve the treatment outcome.

Well stabilized syndesmosis by a cortical screw may affect ankle function [35]. Therefore, the screw is advised to be removed after ligament injuries are healed. In the literature, the time of screw removal is reversely correlated to the incidence of re-diastasis of the syndesmosis [21]. However, late removal of the screw will increase the incidence of screw breakage. In the present study, two cases with screw breakage had no screw removal early. In the literature, screw breakage may have a better ankle function despite that it is not removed $[21,36,37]$. Accordingly, too early removal of the syndesmotic screw is unreasonable. To prevent screw breakage and syndesmotic re-diastasis, TR insertion of the screw with late removal (> 3 months) may be the better choice. The tibiofibular synostosis is generally minimal and TR screw insertion will not hinder the ankle function $[27,38]$.

In 2014, Verim et al. performed a study of finite elemental analysis [39]. The syndesmotic screw was placed at six different altitudes above the plafond. The loads were applied from the tibial plateau and found that the proximal screw insertion had the least syndesmosis widening. They then concluded the best placement of syndesmotic screw to be $30-40 \mathrm{~mm}$ above the plafond. This conclusion is completely contradicted to our concept. Practically, the study of Verim et al. applied loads on the tibial plateau and observed the screw reaction. In fact, the loads should be applied from the plafond because it is the talus pushing the lateral malleolus postero-laterally at the stance phase [33]. In other words, the lower screw nearing the point of force application (i.e. lateral malleolus) can obtain the least loading moment. The difference between Verim et al. and our studies is the different point of force application. Clinically, our interpretation should be more reasonable. Although various favored screw locations had been reported in the literature, none had explained the reasons clearly (Table 2) [21,24,36,39-44].

\section{Table 2}

Favored screw location for stabilization of syndesmotic diastasis

Reported studies Level proximal to plafond (cm) Study modes

Stiehl $^{40} \quad 1990$




\begin{tabular}{|c|c|c|c|}
\hline Xenos $^{41}$ & 1995 & $2.5-4$ & Cadaveric test \\
\hline Hovis $^{42}$ & 2002 & $1-2$ & Case series \\
\hline Beumer $^{43}$ & 2005 & 2 & Cadaveric test \\
\hline Nousiainen $^{44}$ & 2008 & 2.5 & Cadaveric test \\
\hline Dattani $^{36}$ & 2008 & $2-5$ & Review article \\
\hline $\mathrm{Hsu}^{21}$ & & $2-3$ & Case series \\
\hline Verim $^{39}$ & 2014 & $3-4$ & FEA study \\
\hline Schepers ${ }^{24}$ & 2014 & $<4$ & Case series \\
\hline Present study & 2020 & $1-2$ & Case series \\
\hline
\end{tabular}

FEA, finite element analysis

The limitations of our study may include followings: (1) Insufficient sample size, short follow-up, and early screw removal let the comparison be insignificant statistically. Clinically, patients who have malleolar fractures with SD and accept syndesmotic screw stabilization are not many. In our study, only 63 cases within the period of 10 years. In other words, two months are necessary to collect one case. The sample sizes needed in the present study are 56 for each group. Therefore, the statistical significance is difficult to achieve. (2) Based on the literature, modes of a stabilizing screw can affect the treatment outcomes but which is the leading role is still inconclusive. Our study chooses screw location for investigation and other parameters (screw diameter, numbers or purchased cortices) may confound the comparison. Finally, no difference in clinical comparison is obtained. Theoretical superiority becomes the cornerstone of treatment recommendation in the present study.

\section{Conclusion}

Malleolar fractures with SD may occur occasionally. The treatment must be early and careful. Delayed or neglected treatment may cause lifelong disabilities. After malleolar fractures are internally stabilized, SD can be treated with one stabilizing screw. The screw had better be inserted at the trans-syndesmotic area and removed late (at least 3 months).

\section{Declarations}

\section{Ethics approval and consent to participate}

This study had been approved by Institutional Review Board of the authors' institution (Chang Gung Memorial Hospital, Linkou Branch, Taiwan) (IRB: 201900950B0).

\section{Consent to publish}

Not applicable. 
Availability of data and materials

The raw data available upon reasonable request from the corresponding author.

\section{Competing interests}

All authors declare that they have no competing interests.

\section{Funding}

No funding was obtained.

\section{Authors' Contributions}

CCW designed the whole study, analyzed data, and wrote the manuscript.

Five other coauthors provided data and helped to analyze data.

\section{Acknowledgements}

None.

\section{References}

1. Warner SJ, Fabricant PD, Garner MR, Schottel PC, Helfet DL, Lorich DG. The measurement and clinical importance of syndesmotic reduction after operative fixation of rotational ankle fractures. $\mathrm{J}$ Bone Joint Surg Am. 2015; 97: 1935-44.

2. McKeown R, Rabiu AR, Ellard DR, Kearney RS. Primary outcome measures used in interventional trials for ankle fractures: a systemic review. BMC Musculoskelet Disord. 2019; 20: 388.

3. Wang C, Ma X, Wang X, Huang J, Zhang C, Chen L. Internal fixation of distal tibiofibular syndesmotic injuries: A systematic review with meta-analysis. Int Orthop. 2013; 37: 1755-63.

4. Van Heest TJ, Lafferty PM. Injuries to the ankle syndesmosis. J Bone Joint Surg Am. 2014; 96: 60313.

5. Norkus SA, Floyd RT. The anatomy and mechanisms of syndesmotic ankle sprains. J Athl Train. 2001; 36: 68-73.

6. Hermans JJ, Beumer A, de Jong TA, Kleinrensink GJ. Anatomy of the distal tibiofibular syndesmosis in adults: A pictorial essay with a multimodality approach. J Anat. 2010; 217: 633-45.

7. Pakarinen H, Flinkkila T, Ohtonen P, Hyvonen P, Lakovaara M, Leppilahti J, Ristiniemi J. Intraoperative assessment of the stability of the distal tibiofibular joint in supination-external rotation injuries of the ankle: Sensitivity, specificity, and reliability of two clinical tests. J Bone Joint Surg Am. 2011; 93 : 2057-61. 
8. Magan A, Golano P, Maffulli N, Khanduja V. Evaluation and management of injuries of the tibiofibular syndesmosis. Br Med Bull. 2014; 111: 101-15.

9. Okanobo H, Khurana B, Sheehan S, Duran-Mendicuti A, Arianjam A, Ledbetter S. Simplified diagnostic algorithm for Lauge-Hansen classification of ankle injuries. Radiographics. 2012; 32: E71-84.

10. Tartaglione JP, Rosenbaum AJ, Abousayed M, DiPreta JA. Classifications in brief: Lauge-Hansen classification of ankle fractures. Clin Orthop Relat Res. 2015; 473: 3323-8.

11. Han SM, Wu TH, Wen JX, Wang Y, Cao L, Wu WJ, Gao BL. Radiographic analysis of adult ankle fractures using combined Danis-Weber and Lauge-Hansen classification systems. Sci Rep. 2020; 10: 7655.

12. Chen B, Chen $C$, Yang Z, Huang P, Dong H, Zeng Z. To compare the efficacy between fixation with tightrope and screw in the treatment of syndesmotic injuries: A meta-analysis. Foot Ankle Surg. 2019; 25: 63-70.

13. Michelson JD, Wright M, Blankstein M. Syndesmotic ankle fractures. J Orthop Trauma. 2018; 32: 104.

14. Klemt C, Prinold JA, Morgans S, Smith SHL, Nolte D, Reilly P, Bull AMJ. Analysis of shoulder compressive and shear forces during functional activities of daily life. Clin Biomech. 2018; 54: 34-41.

15. Gustilo RB, Anderson JT. Prevention of infection in the treatment of one thousand and twenty-five open fractures of long bones: Retrospective and prospective analyses. J Bone Joint Surg Am. 1976; 58: 453-8.

16. Summers HD, Sinclair MK, Stover MD. A reliable method for intraoperative evaluation of syndesmotic reduction. J Orthop Trauma. 2013; 27: 196-200.

17. Wu CC. Is clinical measurement of anatomic axis of the femur adequate? Acta Orthop. 2017; 88: 40710.

18. Phisitkul P, Ebinger T, Goetz J, Vaseenon T, Marsh JL. Forceps reduction of the syndesmosis in rotational ankle fractures: A cadaveric study. J Bone Joint Surg Am. 2012; 94: 2256-61.

19. Wu CC. Combined tibial lengthening and ankle arthrodesis for patients with certain type of sequelae of poliomyelitis. J Orthop Surg (Hong Kong). 2017; 25: 1-7.

20. Nair AV, Shamsuddin K, John PS, Hamalainen JA, Kurien MA. Correlation of visual analogue scale foot and ankle (VAS-FA) to AOFAS score in malleolar fractures using Indian language questionnaire. Foot Ankle Surg. 2015; 21: 125-31.

21. Hsu YT, Wu CC, Lee WC, Fan KF, Tseng IC, Lee PC. Surgical treatment of syndesmotic diastasis: Emphasis on effect of syndesmotic screw on ankle function. Int Orthop. 2011; 35: 359-64.

22. Wu CC, Shih CH, Lee MY, Tai CL. Biomechanical analysis of location of lag screw of a dynamic hip screw in treatment of unstable intertrochanteric fracture. J Trauma. 1996; 41: 699-702.

23. Wang C, Yang J, Wang S, Ma X, Wang X, Huang J, Zhang C, Chen L, Xu J, Geng X, Wang K. Threedimensional motions of distal syndesmosis during walking. J Orthop Surg Res. 2015; 10: 166. 
24. Schepers $T$, van der Linden $H$, van Lieshout EM, Niesten DD. Technical aspects of the syndesmotic screw and their effect on functional outcome following acute distal tibiofibular syndesmosis injury. Injury. 2014; 45: 775-9.

25. Peek AC, Fitzgerald CE, Charalambides C. Syndesmosis screws: How many, what diameters, where and should they be removed? A literature review. Injury. 2014; 45: 1262-7.

26. Solomonow-Avnon D, Herman A, Wolf A. Mechanism of reducing knee adduction moment by shortening of the knee lever arm via medio-lateral manipulation of foot center of pressure: A pilot study. J Biomech. 2019; 83: 143-9.

27. Kukreti S, Faraj A, Miles JN. Does position of syndesmotic screw affect functional and radiological outcome in ankle fractures? Injury. 2005; 36: 1121-4.

28. Fanter NJ, Inouye SE, McBryde AM Jr. Safety of ankle trans-syndesmotic fixation. Foot Ankle Int. 2010; 31: 433-40.

29. Mroz I, Bachul PJ, Tomaszewski KA, Bereza T, Gil K, Walocha JA, Pasternak A. Classification system of the tibiofibular syndesmosis blood supply and its clinical relevance. Sci Rep. 2018; 8: 10507.

30. Sagi HC, Shah AR, Sanders RW. The functional consequence of syndesmotic joint malreduction at a minimum 2-year follow-up. J Orthop Trauma. 2012; 26: 439-43.

31. van Zuuren WJ, Schepers T, Beumer A, Sierevelt I, van Noort A, van den Bekerom MPJ. Acute syndesmotic instability in ankle fractures: A review. Foot Ankle Surg. 2017; 23: 135-41.

32. van den Heuvel SB, Dingemans SA, Gardenbroek TJ, Schepers T. Assessing quality of syndesmotic reduction in surgically treated acute syndesmotic injuries: A systematic review. J Foot Ankle Surg. 2019; 58: 144-50.

33. Sasse M, Nigg BM, Stefanyshyn DJ. Tibiotalar motion- Effect of fibular displacement and deltoid ligament transection: In vitro study. Foot Ankle Int. 1999; 20: 733-7.

34. Hamid N, Loeffler BJ, Braddy W, Kellam JF, Cohen BE, Bosse MJ. Outcome after fixation of ankle fractures with an injury to the syndesmosis: The effect of the syndesmosis screw. J Bone Joint Surg Br. 2009; 91: 1069-73.

35. Miller AN, Paul O, Boraiah S, Parker RJ, Helfet DL, Lorich DG. Functional outcomes after syndesmotic screw fixation and removal. J Orthop Trauma. 2010; 24: 12-6.

36. Dattani R, Patnaik S, Kantak A, Srikanth B, Selvan TP. Injuries to the tibiofibular syndesmosis. J Bone Joint Surg Br. 2008; 90: 405-10.

37. Dingemans SA, Birnie MFN, Sanders FRK, van den Bekerom MPJ, Backes M, van Beeck E, et al. Routine versus on demand removal of the syndesmotic screw: a protocol for an international randomized controlled trial (RODEO-trial). BMC Musculoskelet Disord. 2018; 19: 35.

38. Droog R, Verhage SM, Hoogendoorn JM. Incidence and clinical relevance of tibiofibular synostosis in fractures of the ankle which have been treated surgically. Bone Joint J. 2015; 97-B: 945-9.

39. Verim O, Er MS, Altinel L, Tasgetiren S. Biomechanical evaluation of syndesmotic screw position: A finite-element analysis. J Orthop Trauma. 2014; 28: 210-5. 
40. Stiehl JB. Complex ankle fracture dislocations with syndesmotic diastasis. Orthop Rev. 1990; 19: 499-507.

41. Xenos JS, Hopkinson WJ, Mulligan ME, Olson EJ, Popovic NA. The tibiofibular syndesmosis. Evaluation of the ligamentous structures, methods of fixation, and radiographic assessment. J Bone Joint Surg Am. 1995; 77: 847-56.

42. Hovis WD, Kaiser BW, Watson JT, Bucholz RW. Treatment of syndesmotic disruptions of the ankle with bioabsorbable screw fixation. J Bone Joint Surg Am. 2002; 84: 26-31.

43. Beumer A, Campo MM, Niesing R, Day J, Kleinrensink GJ, Swierstra BA. Screw fixation of the syndesmosis: A cadaver model comparing stainless steel and titanium screws and three and four cortical fixation. Injury. 2005; 36: 60-4.

44. Nousiainen MT, McConnell AJ, Zdero R, McKee MD, Bhandari M, Schemitsch EH. The influence of the number of cortices of screw purchase and ankle position in Weber $\mathrm{C}$ ankle fracture fixation. $\mathrm{J}$ Orthop Trauma. 2008; 22: 473-8.

\section{Figures}

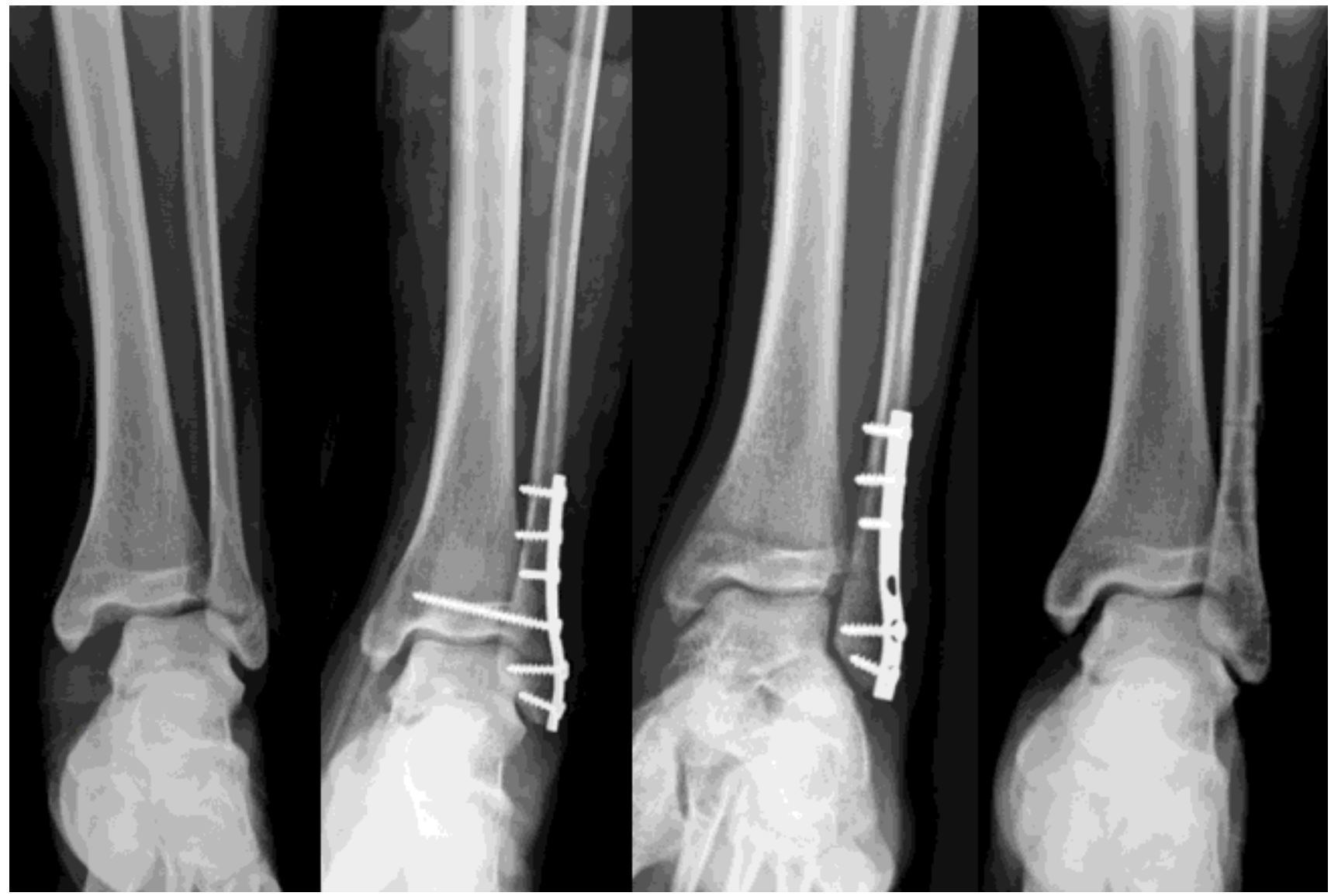

Figure 1

A 30-yr-old man sustained a left stage 4 supination-external rotation ankle injury due to motorcycle accident. Fibular plating with a trans-syndesmotic screw was inserted. The syndesmotic screw was 
removed at 2 months. A good ankle function was achieved at 1.8-year follow-up.

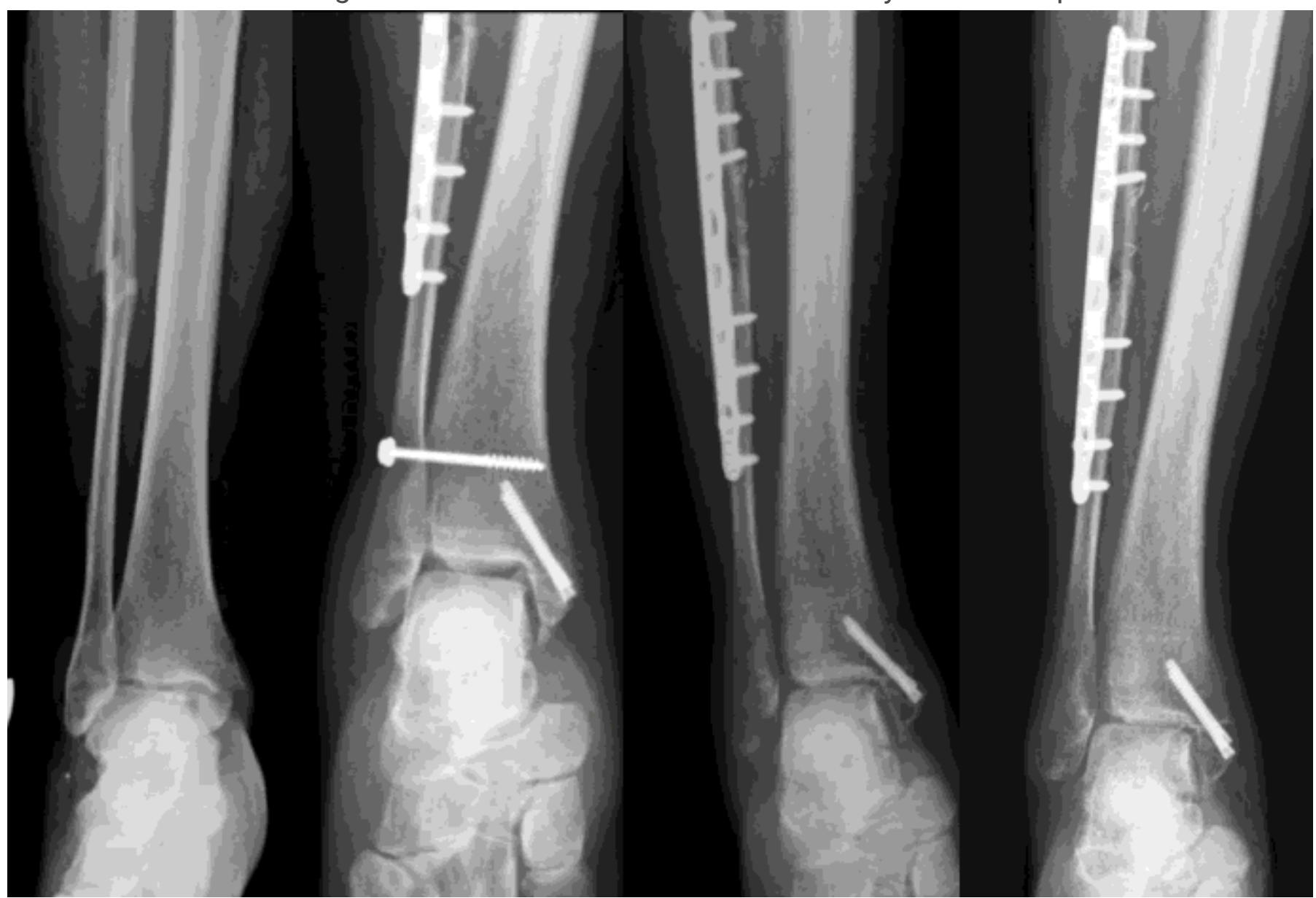

Figure 2

A 20-yr-old man sustained a right stage 4 pronation-external rotation ankle injury due to motorcycle accident. Fibular plating with a supra-syndesmotic screw was inserted. The syndesmotic screw was removed at 2.5 moths. A good ankle function was achieved at 1.2-year follow-up. 

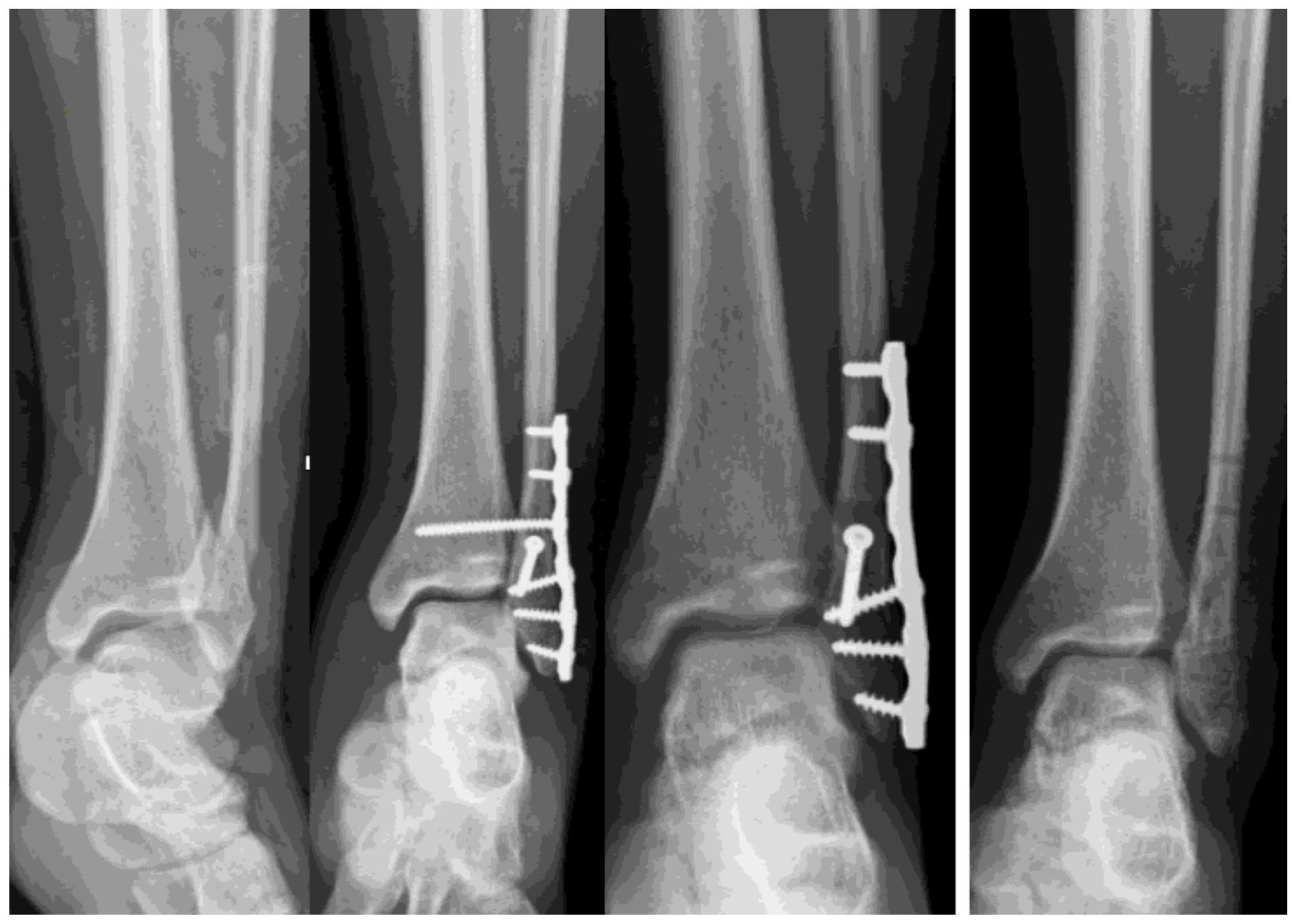

\section{Figure 3}

An 18-yr-old woman sustained a left stage 4 supination-external rotation ankle injury due to slide. Fibular plating with a trans-syndesmotic screw was inserted. The syndesmotic screw was removed at 1.5 months and re-diastasis occurred. A good ankle function was achieved at 1.6-year follow-up. 


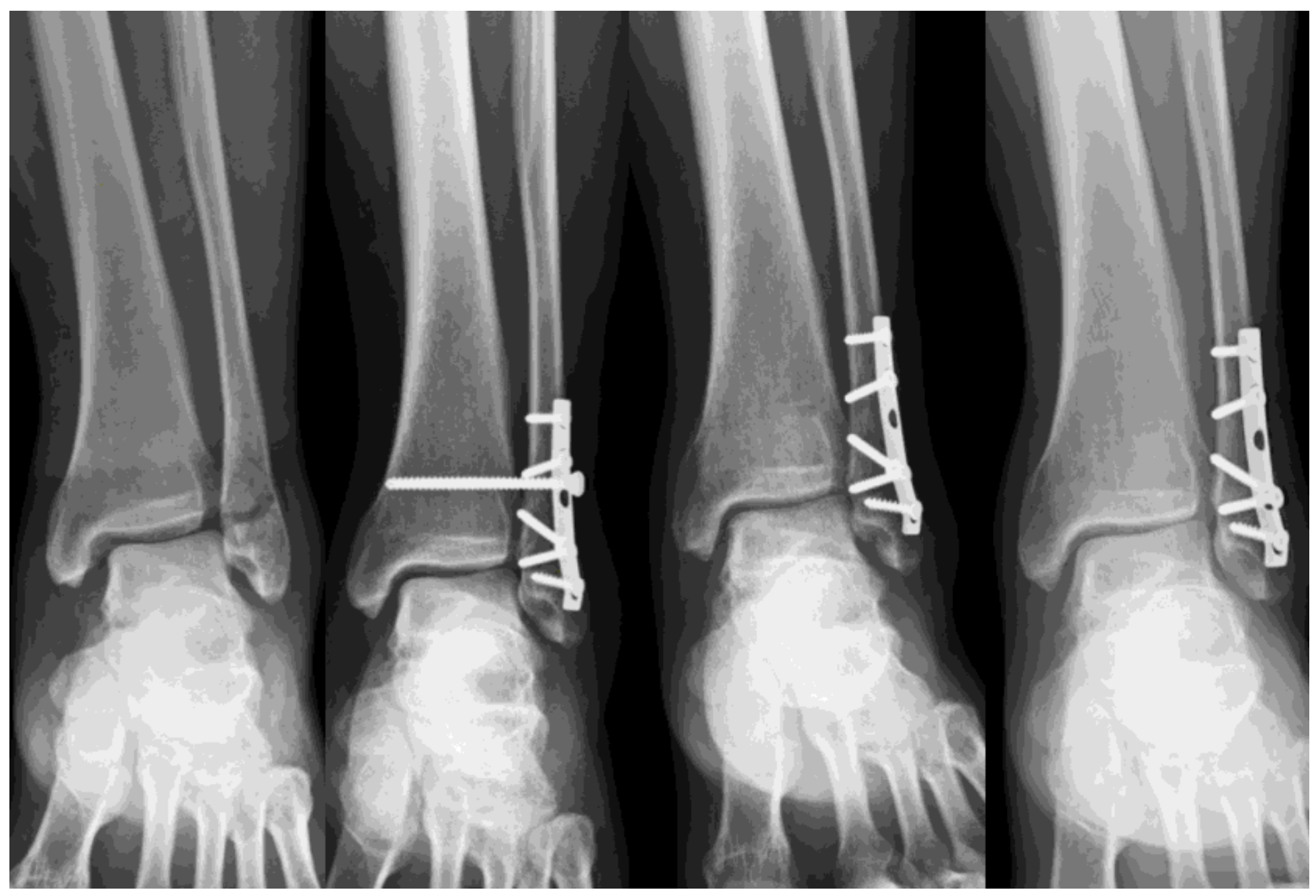

Figure 4

A 39-yr-old man sustained a left stage 4 supination-external rotation ankle injury due to slide. Fibular plating with one supra-syndesmotic screw was inserted. The syndesmotic screw was removed at 1.5 months and re-diastasis occurred. A good ankle function was achieved at 1-year follow-up. 


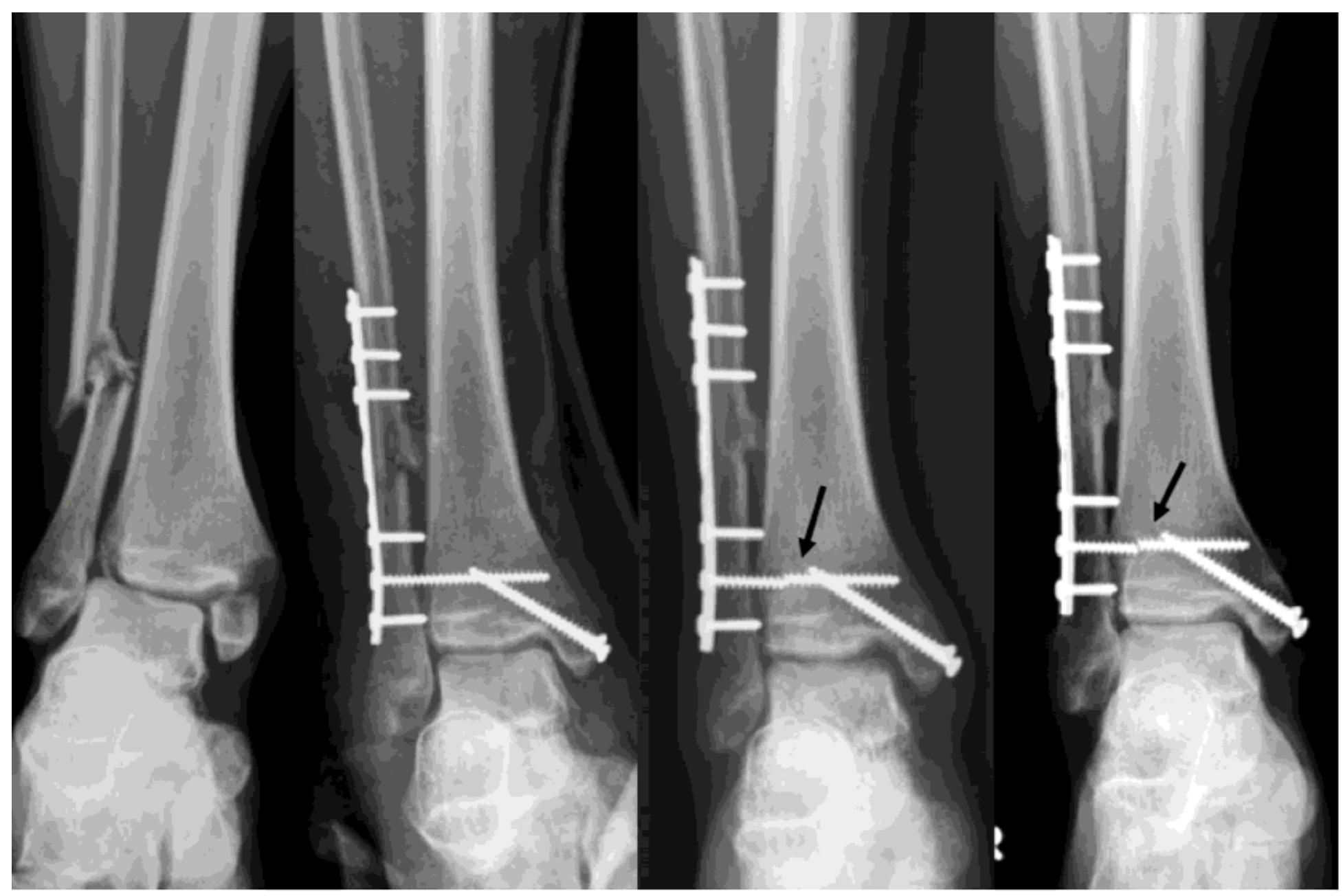

Figure 5

A 20-yr-old man sustained a right stage 3 pronation-external rotation ankle injury due to motorcycle accident. Screwing and plating were inserted for bimalleolar fractures and a trans-syndesmotic screw was inserted for syndesmotic diastasis. The syndesmotic screw broke (arrow) at 6 months without removal. A good ankle function was achieved at 1.5-year follow-up. 


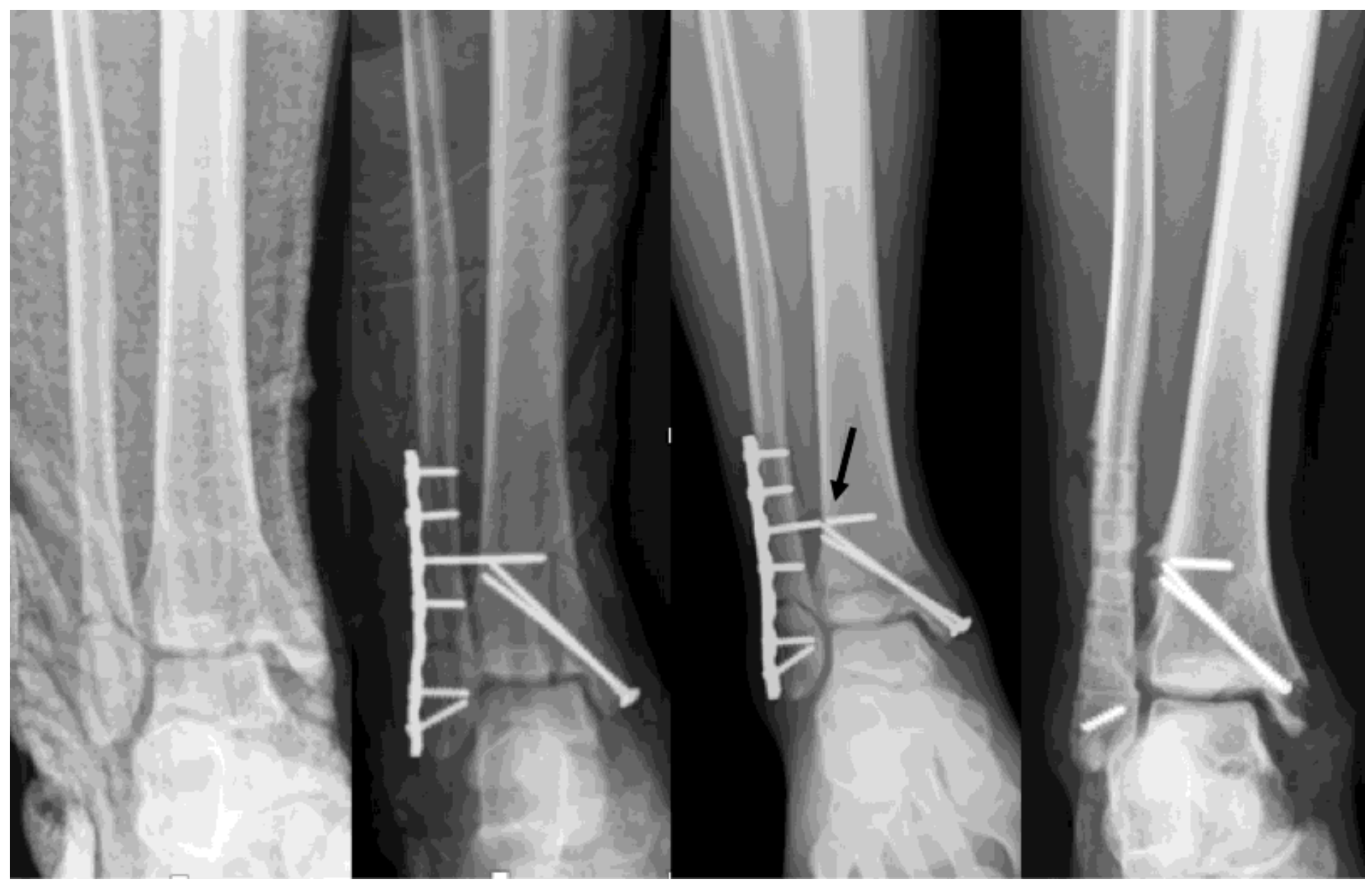

\section{Figure 6}

A 21-yr-old man sustained a right stage 4 supination-external rotation ankle injury due to fall from stairs. Screwing and plating were inserted for bimalleolar fractures and a supra-syndesmotic screw was inserted for syndesmotic diastasis. The syndesmotic screw broke (arrow) at 1.5 months without early removal and re-diastasis of syndesmosis occurred. A fair ankle function (AOFAS score of 76 points) was achieved at 2-year follow-up. 